\title{
One-step high-throughput assay for quantitative detection of $\beta$-galactosidase activity in intact Gram-negative bacteria, yeast, and mammalian cells
}

\author{
Faustino Vidal-Aroca, Michele Giannattasio, Elisa Brunelli, Alessandro \\ Vezzoli, Paolo Plevani, Marco Muzi-Falconi, and Giovanni Bertoni \\ Università degli Studi di Milano, Milano, Italy
}

BioTechniques 40:433-440 (April 2006)

doi $10.2144 / 000112145$

The capability to monitor gene expression represents an invaluable tool to understand cell regulation. The activity of $\beta$-galactosidase ( $\beta$-gal), the product of the lacZ gene of Escherichia coli, is one of the most widely used reporters of gene expression. $\beta-G a l$ is a versatile reporter since its activity can be monitored using a variety of chromogenic and fluorogenic substrates in both prokaryotes and eukaryotes. However, efficient access of these substrates to $\beta$-gal enzyme is hindered by cellsurface barriers. Routine protocols for the quantitative determination of the in vivo expression of $\beta$-gal activity in any system require cell permeabilization or destruction of cell integrity to obtain crude extracts (1-8). This makes $\beta$-gal determination less straightforward than, for instance, green fluorescent protein (GFP)- or luciferase-based reporter systems, which do not require cell lysis or permeabilization. In general, besides being time- and material-consuming, multiple step manipulations before the addition of the $\beta$-gal substrate can hinder the development of highthroughput protocols, which are essential in genome-wide approaches. It would be desirable to develop simple one-step assays that could be automated for parallel screening of large numbers of samples. In this work, we report a fast method, functional in a broad range of organims, which makes $\beta$-gal determination as straightforward as other reporter systems mentioned here. In this system, $\beta$-gal activity is determined through the simple addition of the fluorogenic substrate 4-methylumbelliferyl $\beta$-D-galactopyranoside
(MUG) $(8,9)$ to intact cells of bacteria, yeast and mammalia. We show that, since it does not require centrifugation or cell lysis steps, this assay can be applied to high-throughput screenings, such as mutagenesis or two-hybrid, based on the differential expression of the lac $Z$ gene.

First, we evaluated MUG as $\beta$-gal substrate for quantitative assays on intact bacterial cells. Bacterial cultures were grown overnight in 96-well microplates, $20 \mu \mathrm{L}$ of each culture were transferred with a Multiprobe ${ }^{\circledR}$ IIEx Robotic Liquid Handling System (Packard BioScience, Meriden, CT, USA) to 96-well microplates (black/ clear Optilux ${ }^{\mathrm{TM}}$ flat bottom; BD Falcon ${ }^{\mathrm{TM}}$; BD Biosciences, San Jose, CA, USA) containing $80 \mu \mathrm{L}$ of Z-buffer (3), and the corresponding cell density was evaluated by determining the $\mathrm{A}_{595}$ in a Model 550 Microplate Reader (Bio-Rad Laboratories, Hercules, CA, USA). Twenty-five microliters 1 $\mathrm{mg} / \mathrm{mL}$ MUG in dimethyl sulfoxide (DMSO) were then added to each well, and the samples were incubated at room temperature for $15 \mathrm{~min}$. The reaction was stopped with $30 \mu \mathrm{L} 1 \mathrm{M}$ $\mathrm{Na}_{2} \mathrm{CO}_{3}$. The amount of fluorescence generated by $\beta$-gal-dependent MUG hydrolysis was quantitated in a microplate fluorometer (FluoroCount ${ }^{\mathrm{TM}}$; Packard BioScience), using as a blank reference the assay on a cell-free culture medium sample. Arbitrary units of $\beta$-gal activity (MUG units) were calculated as follows:

$$
\frac{\mathrm{F}_{360 / 460}}{\mathrm{t} \times \mathrm{A}_{595}}
$$

where $\mathrm{F}_{360 / 460}$, $\mathrm{t}$, and $\mathrm{A}_{595}$ are sample fluorescence at the end of the reaction, time of reaction in minutes, and absorbance of the cell suspension, respectively.

As shown in Figure 1A, we examined a selection of both $E$. coli (ER100) and Pseudomonas putida (MAD1, MAD2) reporter strains carrying a chromosomal fusion of the lacZ gene with the $P$. putida $P u$ promoter activated by the toluene-responsive $\mathrm{XylR}$ regulator $(10,11)$, and expressing a wide range of $\beta$-gal activities. In MAD1, which contains the wild-type XylR regulator, we detected a strong 70- to 150-fold stimulation of $P u$ activity upon toluene addition. In MAD2, we observed the constitutively high $P u$ activity mediated by the XylR $\Delta$ A variant. In $E$. coli ER100, where XylR was expressed from a plasmid, we detected a higher basal level of $P u$ expression that increased approximately 2.5-fold upon toluene addition. Importantly, strains isogenic to those described here either lacking the $P u$-lacZ fusion (ER99 and KT2442) or the activator gene (ET99) showed negligible fluorescence upon MUG addition (Figure 1A), indicating that the majority of fluorescence in the assay derived from MUG hydrolysis by $\beta$-gal. Furthermore, MAD2 cells showed high $\beta$-gal activity even in the absence of toluene, indicating that the increase in $\beta$-gal expression after toluene addition in ER100 and MAD1 was not an artifact due to cell permeabilization by toluene. This was also confirmed by the 2.5-fold induction of $P u$ expression upon 2-methylphenol addition to $E$. coli ET100 (Figure 1A), a strain expressing the XylR-related and methylphenol-responsive activator TouR (12). Thus, the $\beta$-gal activity assay based on MUG hydrolysis is an effective method for measuring reporter gene activity in bacteria without the need for a cell lysis step. The reproducibility and sensitivity of this in vivo assay is at least as good as the one displayed by the classic Miller's test (see Supplementary Figure S1 available online at www.BioTechniques.com).

We also validated the use of the MUG assay in a high-throughput expression screen in bacteria by determining TouR transcriptional induction 
Figure 1. Measurement of $\beta$-gal activity by MUG assay in intact Escherichia coli and Pseudomonas putida strains. (A) E. coli ER99 and ER100 are CC118 (17) and CC118 Pu-lacZ (11) derivatives, respectively, carrying the medium copy-number plasmid $\mathrm{pACYC} x y l R$, a $\mathrm{pA}$ CYC184-based (18) plasmid expressing the $x y l R$ gene under the control of its native promoter. $E$. coli ET99 and ET100 are CC118 Pu-lacZ carrying pTrc99a (19) and pTRR6N-touR, a pTrc99abased plasmid expressing a $6 \times$ His-tagged TouR protein (12), respectively. $P$. putida MAD1 and MAD2 are KT2442 (14) reporter derivatives bearing a hybrid mini-Tn5 transposon that includes a transcriptional $P u$-lacZ fusion along with the sequence of either the wild-type $x y l R$ gene or the $x y l R$ allele encoding the constitutively active $\mathrm{XylR} \Delta \mathrm{A}$ protein, respectively (10). The MUG assay was performed on cells of the indicated strains that had been grown overnight, both in the absence (empty bars) and the presence (shaded bars) of effector molecules [toluene vapors for XylR- or $1 \mathrm{mM}$ 2-methylphenol (2-mp) for TouR-expressing strains], in 96-well microplates filled with $100 \mu \mathrm{L}$ LB medium, supplemented with either ampicillin for ET99 and ET100 or chloramphenicol for ER99 and ER100, or $100 \mu \mathrm{L}$ synthetic mineral M9 medium supplemented with $0.2 \%$ citrate, in the case of both MAD1 and MAD2. $\beta$-Gal activity is reported as MUG units, and the value for each strain represents the average of three MUG assays performed on eight independent cultures. (B) Variability in the TouR-mediated activation of transcription within a PSM library generated as described previously $(13,20)$. The MUG assay was performed, in two independent experiments, on 96 PSM-derived ET100 clones grown overnight in 96-well microplates filled with $100 \mu \mathrm{L}$ LB medium supplemented with ampicillin, both in the presence and the absence of $1 \mathrm{mM} 2$-methylphenol, the TouR effector molecule. For each clone in both conditions, values represent the ratio between the average MUG units determined in the two experiments, and the average MUG units expressed by ET100 carrying a nonmutagenized

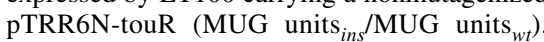
MUG, 4-methylumbelliferyl $\beta$-D-galactopyranoside; LB, Luria Bertani; PSM, pentapeptide scanning mutagenesis.

activity in a collection of touR mutants. We applied pentapeptide scanning mutagenesis (PSM) (13) to the TouRexpressing plasmid pTRR6N-touR and evaluated the expected variability in TouR activity in a large pool of random $15 \mathrm{bp}$ insertions, both within the touR gene and in the vector backbone. Briefly, 96 clones of the pool were inoculated in a 96-well microplate and grown in the presence and the absence of 2-methylphenol. For each sample, $P u$ promoter induction was analyzed by high-throughput automated MUG assay and compared with the $P u$ performance in the reference E. coli ET100 carrying

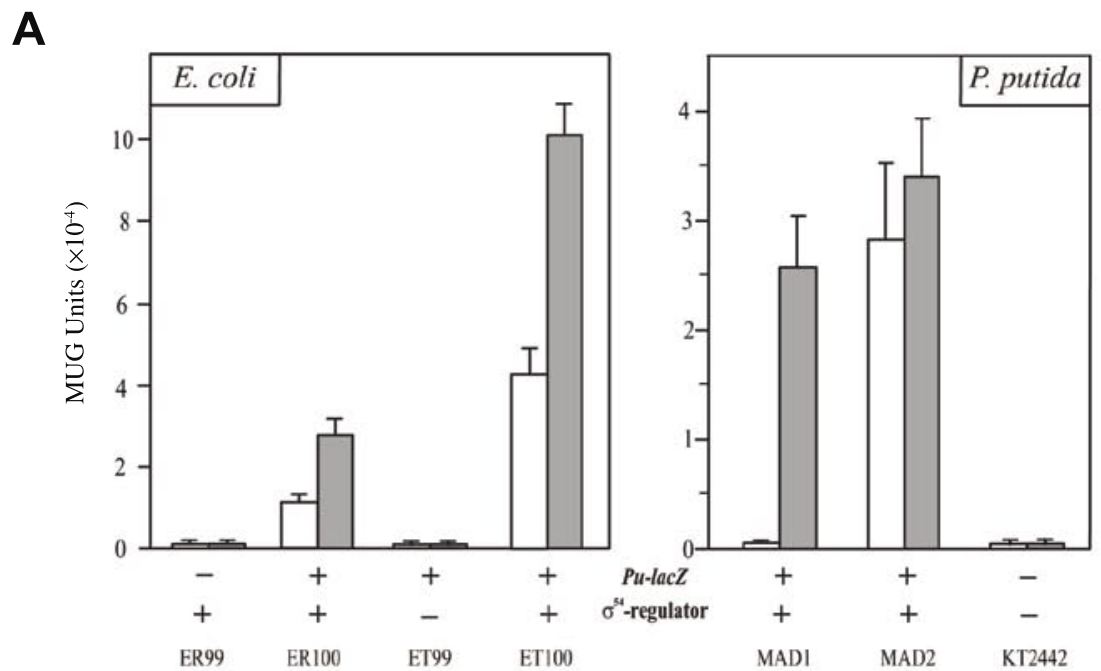

B

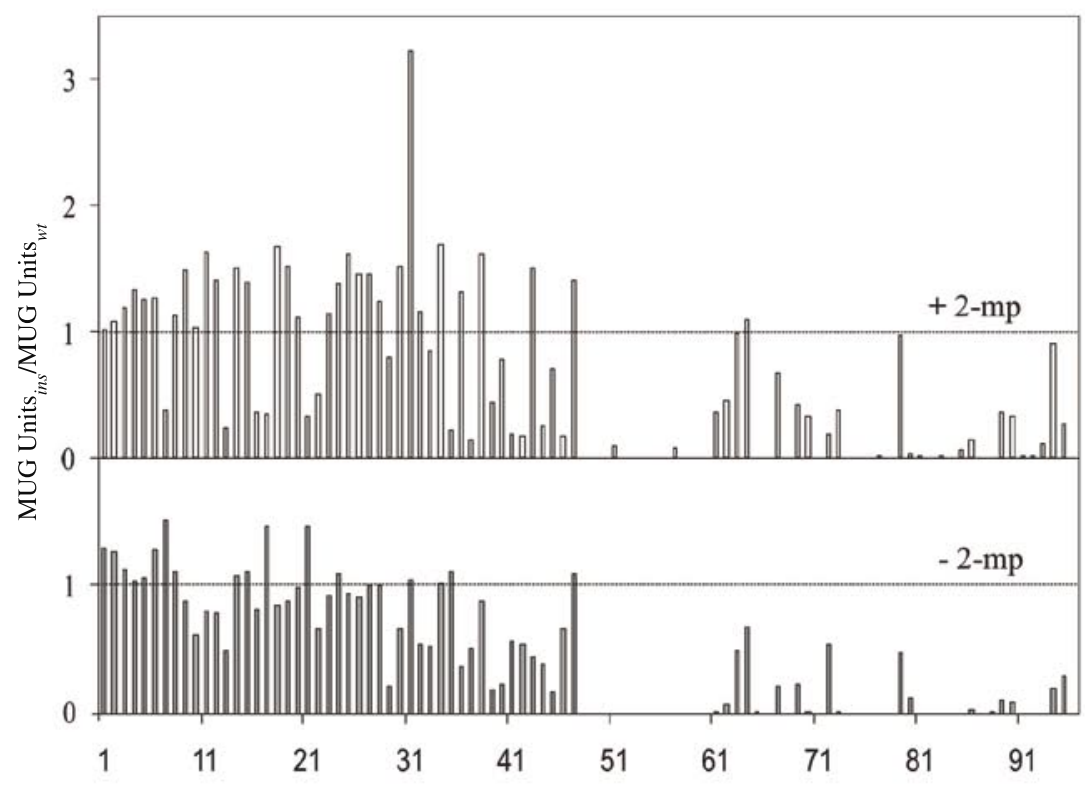

a nonmutagenized pTRR6N-touR. As shown in Figure 1B, the collection of 96 clones derived from the mutagenesis showed a high degree of variability in TouR-mediated activation of $P u$. The site of the 15-bp insertion was mapped in every clone. All clones that showed a significant deviation from the TouRmediated activation pattern of the reference also contained an insertion within the touR gene. By contrast, the majority of the clones showing an activation pattern similar to the control strain had the 15-bp insertion in the vector backbone. Only three clones containing an insertion within the touR gene exhibited no significant deviation from the reference in the MUG assay, possibly because the mutation occurred in TouR regions tolerant for the insertion. A reexamination of the mutated clones with Miller's assay confirmed the results obtained by the MUG assay for each clone tested (F. Vidal-Aroca and G. Bertoni, unpublished results).

We also assessed the utility of the MUG assay in a promoter-trapping experiment in $P$. putida KT2442, intended to identify toluene-responsive promoters. First, a library of KT2442 insertion mutants was generated by 
delivery of the minitransposon miniTn5 lacZ (14). Approximately 3000 clones were inoculated into 96-well microplates and grown in the absence and in the presence of toluene. $\beta-G a l$ activity of every clone in each growth condition was determined by the automated high-throughput MUG assay. About 250 clones showing a ratio of at least 2.5 between $\beta$-gal activity in the presence versus the absence of toluene were arrayed in 96-well microplates, and their $\beta$-gal activity was retested by the MUG assay in three independent experiments. Two clones showed a significant induction ratio of 3 and 20, respectively. These results were also confirmed by Miller's assay (E. Brunelli and G. Bertoni, unpublished results) and validate the MUG assay as a very useful method for the fast screening of large numbers of samples, allowing several repetitions of the experiment to obtain statistically significant data.

We next examined the possibility to extend the MUG assay described here to yeast by using a reporter strain (EGY48) harboring the positive and negative control plasmids belonging to the MATCHMAKER LexA TwoHybrid System (Clontech, Mountain View, CA, USA). In particular, we compared the $\beta$-gal activity of EGY48[p8op-lacZ] strains carrying either the empty vectors pLexA and pB42AD or pLexA-53 and pB42AD-T, in which the interactors p53 and simian virus 40 (SV40) T-antigen were cloned as hybrid proteins, respectively. In this system, prey fusion protein expression is under the control of the yeast galactose-inducible GAL1 promoter. We assayed intact yeast cells grown both in the presence and the absence of galactose. As shown in Figure 2A, the results obtained in yeast faithfully reflect what is expected for these control plasmids (see Supplementary Figure S1 for a direct comparison with a traditional $\beta$-gal assay in yeast). In the MUG assay, the pLexA/pB42AD pair showed barely detectable $\beta$-gal activity both in the presence and the absence of galactose. By contrast, the pLexA-53/ pB42AD-T containing strain displayed a strong $\beta$-gal induction by galactose (about 30-fold), traceable to T-antigen overexpression. To test whether another fluorogenic substrate of $\beta$-gal could be used in place of MUG, we also performed the experiments with fluorescein di- $\beta$-D-galactopyranoside (FDG). We added $25 \mu \mathrm{L}$ of a $1 \mathrm{mg} / \mathrm{mL}$ solution of FDG to yeast cell suspensions in a 96-well microtiter plate, incubated at room temperature for 15 min, and stopped the reaction with the addition of $30 \mu \mathrm{L} 1 \mathrm{M} \mathrm{Na}_{2} \mathrm{CO}_{3}$ as in the
MUG assay. Equivalent results were obtained in the FDG assay (Figure $2 \mathrm{~A}$ ), suggesting that both substrates can be effectively applied to growing yeast cultures without requiring prior collection of cell pellets and production of crude protein extracts. Since this is a rate-limiting step in screening large numbers of samples, we extended the MUG assay to an automated high-

\section{A}

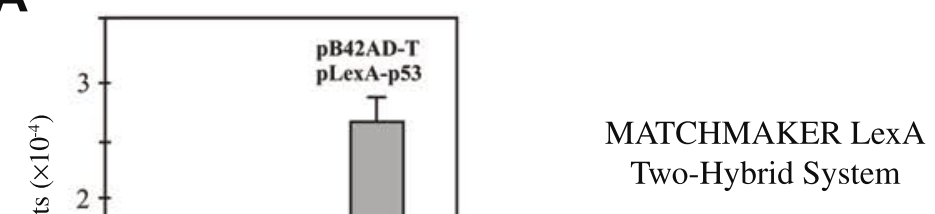

Two-Hybrid System

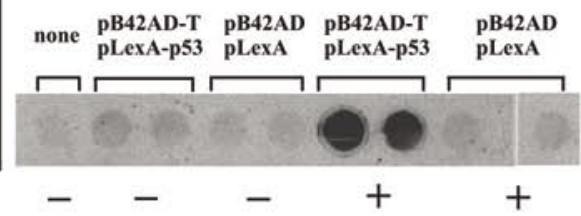

B

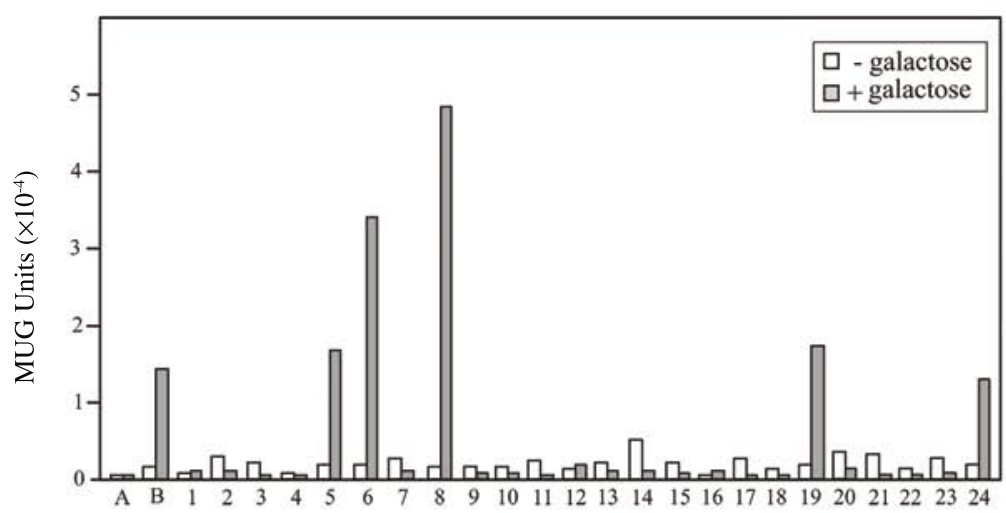

Figure 2. Measurement of $\beta$-gal activity by MUG assay in intact yeast cells. (A) (Left) The MUG assay was performed on the EGY48[p8op-lacZ] host strain $(21,22)$, carrying the indicated plasmid pairs, pLexA and pB42AD (21), pLexA-53 and pB42AD-T (MATCHMAKER LexA Two-Hybrid System) grown overnight in 96-well microplates filled with $100 \mu \mathrm{L}$ SD medium both in the presence (shaded bars) and the absence (empty bars) of $2 \%$ galactose. $\beta$-gal activity is reported as MUG units, and the value for each strain represents the average of at least three MUG assays performed on eight independent microcultures. (Right) To measure $\beta$-gal activity in the same yeast strains, using FDG instead of MUG, FDG was added to the cell suspensions in black, clear-bottom 96-well microplates. The amount of fluorescence arising from FDG hydrolysis by $\beta$-gal was determined by the scanning of the well bottoms in a Typhoon $^{\mathrm{TM}} 8600$ variable mode Imager (GE Healthcare BioSciences, Piscataway, NJ, USA) setting the excitation and emission at $532 \mathrm{~nm}$ and 526SP, respectively. (B) MUG assay as a tool for high-throughput two-hybrid screens. Among approximately 800 clones that were positive by auxotrophic selection from a two hybrid screen, 24 clones were tested by MUG assay. (A) Negative control: cells harboring bait and prey cloning vectors. (B) Positive control: cells expressing as bait the Ddc1 DNA damage checkpoint protein and as prey the Rad24 clamp loader subunit. Cells were transferred from the selection plate to a 96-well microplate containing $100 \mu \mathrm{L}$ SD medium, in the presence (shaded bars) or absence (empty bars) of $2 \%$ galactose, and the MUG assay was performed. $\beta$-gal activity is reported as MUG units, and the value for each strain represents the average of at least three MUG assays performed on eight independent microcultures. MUG, 4-methylumbelliferyl $\beta$-D-galactopyranoside; FDG, fluorescein di$\beta$-D-galactopyranoside. 
throughput screening in intact yeast cells. Yeast diploid clones $\left(1.5 \times 10^{7}\right)$, derived from a mating-based twohybrid screening using the yeast checkpoint protein Ddc1 as bait (15), were selected in the presence of galactose on -LEU medium, which allows growth only if bait and prey interact. Twentyfour of the approximately 800 positive clones were grown in a 96-well microplate, in the absence and the presence of galactose, and the automated MUG assay was performed. Figure 2B shows the MUG units measured in the 24 cultures in the absence or presence of galactose. Clone numbers 5, 6, 8, 19 , and 24 were scored as positives in this interaction screening assay since the $\beta$-gal activity was dependent upon galactose induction. The application of the MUG assay to such a screen is of notable value because many of the clones that were positive in the auxotrophic screen revealed very low $\beta$-gal expression. Thus, this method allows users to reduce rapidly the number of clones to be analyzed after the first round of selection and also provides a quick indication of the most promising clones based on the strength of the interaction between bait and prey. All the results shown in Figure 2B were confirmed by Miller's test in yeast cell extracts (data not shown). It is worth reporting that clone number 8 , the strongest Ddc1 interactor, corresponds to the $\operatorname{Rad} 14$ repair factor, and clone number 6 was Rev7, a subunit of a translesion DNA synthesis polymerase. Both these factors have been shown to interact physically and functionally with Ddc1 $(15,16)$. The setup we describe allows for rapid screening of numerous strains in different experimental conditions.

To rule out that the MUG assay detected $\beta$-gal released from cells during either growth or exposure to DMSO (in which MUG was dissolved), we performed the MUG assay for both bacteria and yeast on $(i)$ culture media from which cells had been removed after growth and (ii) samples of cleared 20\% DMSO in Z-buffer used to treat cells for $30 \mathrm{~min}$. In all cases, fluorescence values of the MUG assay were undistinguishable from a blank reference.
Finally, we tested the MUG assay on intact mammalian cells. To this end, adherent NIH 3T3 mouse fibroblasts, either nontransfected or transfected with pSV- $\beta$-Galactosidase Control Vector (Promega, Madison, WI, USA), carrying lacZ under the control of SV40 early promoter, were recovered after gentle treatment with trypsin, resuspended in Z-buffer at 500 cells $/ \mu \mathrm{L}$, and $100 \mu \mathrm{L}$ aliquots were distributed in black, clearbottom 96-well microplates $(50,000$ cells/well). The determination of the number of viable cells and the cellular density were estimated by Trypan blue exclusion assay. Twenty-five microliters of $1 \mathrm{mg} / \mathrm{mL}$ MUG in DMSO were added to each cell suspension. Samples were incubated at room temperature for $60 \mathrm{~min}$, and $30 \mu \mathrm{L} 1 \mathrm{M} \mathrm{Na}_{2} \mathrm{CO}_{3}$ were added to stop the reaction. The average of fluorescence emission by $\beta$-galhydrolyzed MUG measured on five wells in three independent experiments for each kind of cells was determined as described above and was 25-fold higher in the wells containing transfected fibroblasts. Remarkably, the mean fluorescence value of the nontransfected mouse fibroblasts was not significantly different from that of control wells containing $100 \mu \mathrm{L} \mathrm{Z}$-buffer only (data not shown).

In summary, we report here that reliable $\beta$-gal determination can be accomplished in a broad range of organisms by the simple addition of MUG without any centrifugation, cell permeabilization, or lysis steps. This single-step procedure, combined with direct determination of $\mathrm{A}_{595}$ and $\mathrm{F}_{360 / 460}$ in the same well, renders the MUG assay a more suitable and economical option for fully automated highthroughput screening. Therefore, it can find large application in fields such as functional genomics, drug discovery, and diagnostic screens, which rely on high-throughput applications.

\section{ACKNOWLEDGMENTS}

This work was partially supported by grants from AIRC, CIB, MIUR, Progetto FIRB-MIUR "Genomica $e$ proteomica nello studio di funzioni cellulari complesse," Progetto FIRBMIUR "Risposta globale a stress am- bientali nei batteri" (RBAU01KHM2), and contract QLK3-CT-2000-00170 of the European Union. We are grateful to $V$. de Lorenzo for helpful discussions, to E. Rescalli, F. Lazzaro, and M. Minuzzo for technical support, and to $J$. Garmendia for providing the plasmid pACYCxylR.

\section{COMPETING INTERESTS STATEMENT}

F.V-A., M.G., P.P., M.M.-M., and G.B. declare that the methods and related applications described were filed with the Italian Patent Office and are the subject of an international patent pending. The remaining authors declare no competing interests.

\section{REFERENCES}

1. Rupp, S. 2002. LacZ assays in yeast. Methods Enzymol. 350:112-131.

2.Mockli, N. and D. Auerbach. 2004. Quantitative $\beta$-galactosidase assay suitable for high-throughput applications in the yeast two-hybrid system. BioTechniques 36:872-876.

3. Miller, J.H. 1972. Experiments in Molecular Genetics. CSH Laboratory Press, Cold Spring Harbor, NY.

4. Thibodeau, S.A., R. Fang, and J.K. Joung. 2004. High-throughput $\beta$-galactosidase assay for bacterial cell-based reporter systems. BioTechniques 36:410-415.

5. Serebriiskii, I.G. and E.A. Golemis. 2000 Uses of lacZ to study gene function: evaluation of $\beta$-galactosidase assays employed in the yeast two-hybrid system. Anal. Biochem. 285:1-15.

6. Serebriiskii, I.G., R. Fang, E. Latypova, R. Hopkins, C. Vinson, J.K. Joung, and E.A. Golemis. 2005. A combined yeast/bacteria two-hybrid system: development and evaluation. Mol. Cell. Proteomics 4:819-826.

7. Eglen, R.M. and R. Singh. 2003. $\beta$-galactosidase enzyme fragment complementation as a novel technology for high throughput screening. Comb. Chem. High Throughput Screen. 6:381-387.

8. Prepiak, P., Z. Chromikova, and I. Barak. 2001. Use of yeast two-hybrid system for detection of Bacillus subtilis FtsZ protein partners. Folia Microbiol. (Praha) 46:292-296.

9. McGuire, J.B., T.J. James, C.J. Imber, S.D. St Peter, P.J. Friend, and R.P. Taylor. 2002. Optimisation of an enzymatic method for $\beta$ galactosidase. Clin. Chim. Acta 326:123-129.

10. Fernández, S., V. de Lorenzo, and J. PérezMartín. 1995. Activation of the transcriptional regulator XylR of Pseudomonas putida by release of repression between functional domains. Mol. Microbiol. 16:205-213.

11.de Lorenzo, V., M. Herrero, M. Metzke, and K.N. Timmis. 1991. An upstream XylR- 
and IHF-induced nucleoprotein complex regulates the sigma 54-dependent Pu promoter of TOL plasmid. EMBO J. 10:1159-1167.

12. Arenghi, F.L., P. Barbieri, G. Bertoni, and V. de Lorenzo. 2001. New insights into the activation of $o$-xylene biodegradation in Pseudomonas stutzeri OX1 by pathway substrates. EMBO Rep. 2:409-414.

13. Hayes, F. and B. Hallet. 2000. Pentapeptide scanning mutagenesis: encouraging old proteins to execute unusual tricks. Trends Microbiol. 8:571-577.

14. de Lorenzo, V. and K.N. Timmis. 1994. Analysis and construction of stable phenotypes in gram-negative bacteria with $\mathrm{Tn} 5$ and Tn10-derived minitransposons. Methods Enzymol. 235:386-405.

15. Giannattasio, M., F. Lazzaro, M.P. Longhese, P. Plevani, and M. Muzi-Falconi. 2004. Physical and functional interactions between nucleotide excision repair and DNA damage checkpoint. EMBO J. 23:429-438.

16. Sabbioneda, S., B.K. Minesinger, M. Giannattasio, P. Plevani, M. Muzi-Falconi, and S. Jinks-Robertson. 2005. The 9-1-1 checkpoint clamp physically interacts with Polzeta and is partially required for spontaneous Polzeta-dependent mutagenesis in Saccharomyces cerevisiae. J. Biol. Chem. 280:38657-38665.

17. Manoil, C. and J. Beckwith. 1985. TnphoA: a transposon probe for protein export signals. Proc. Natl. Acad. Sci. USA 82:8129-8133.

18. Chang, A.C. and S.N. Cohen. 1978. Construction and characterization of amplifiable multicopy DNA cloning vehicles derived from the P15A cryptic miniplasmid. J. Bacteriol. 134:1141-1156.

19. Amann, E., B. Ochs, and K.J. Abel. 1988. Tightly regulated tac promoter vectors useful for the expression of unfused and fused proteins in Escherichia coli. Gene 69:301-315.

20. Hallet, B., D.J. Sherratt, and F. Hayes. 1997. Pentapeptide scanning mutagenesis: random insertion of a variable five amino acid cassette in a target protein. Nucleic Acids Res. 25:1866-1867.

21. Gyuris, J., E. Golemis, H. Chertkov, and R. Brent. 1993. Cdi1, a human G1 and S phase protein phosphatase that associates with Cdk2. Cell 75:791-803.

22. Estojak, J., R. Brent, and E.A. Golemis. 1995. Correlation of two-hybrid affinity data with in vitro measurements. Mol. Cell. Biol. 15:5820-5829.

Received 27 October 2005; accepted 22 January 2006.

Address correspondence to Giovanni Bertoni, Dipartimento di Scienze Biomolecolari e Biotecnologie, Università degli Studi di Milano, Via Celoria 26, 20133, Milan, Italy. e-mail: giovanni.bertoni@unimi.it

To purchase reprints

of this article, contact 